\title{
A Study of Cost Function Selection in Model Predictive Control Applications
}

\author{
Diego Rojas, Marco Rivera, Patrick Wheeler, Pericle Zanchetta, Galina Mirzaeva and Jaime Rohten
}

\begin{abstract}
The cost function selection is considered one of the most relevant aspects for the implementation of Model Predictive Control strategies. In this paper a study of the most common cost functions used for the control of a two level voltage source inverter is presented. The paper introduces several cost function alternatives that could be considered for different power electronic converter applications to compare the implementation and resulting converter waveforms. The results show that Model Predictive Control is an alternative for the implementation of different control objectives in power electronic converters.

Keywords-Cost function selection, model predictive control, voltage source inverter
\end{abstract}

\section{INTRODUCCIÓN}

La función de costo es fundamental para el desarrollo de un control predictivo, por lo tanto el diseño dependerá de los requerimientos de control en un convertidor de potencia. Una de las principales características del control predictivo es que puede controlar múltiples objetivos, debido a que se puede agregar condiciones y términos adicionales en la función de costo. Últimamente, se han desarrollado varias funciones de costo para distintos requerimientos en los convertidores de potencia, tal como control de los vectores de voltaje en coordenadas $d q$ [1], control de flujo del estator [2], control de la potencia reactiva [3], control de la corriente en coordenadas $d q$ [4], entre otros. En el siguiente paper se presentan distintas formas de implementar la función de costo y distintos términos usados para evaluar diferentes requerimientos u objetivos de control. Además, se presentan los resultados simulados en Matlab/Simulink aplicando varias funciones de costos en un inversor fuente voltaje trifásico para diferentes requerimientos.

\section{Modelo Matemático del InVERsor Fuente de VOLTAJE}

La topología del bien conocido inversor fuente de voltaje (VSI) está detallado en la Fig. 1. Una restricción para la correcta operación de este convertidor es asegurar que los dos switches de cada pierna deben operar en un modo complementario con el fin de evitar cortocircuitos de la fuente DC. Como

Diego Rojas. Estudiante del Doctorado en Sistemas de Ingeniería, Facultad de Ingeniería, Universidad de Talca, Curicó, Chile.

Marco Rivera. Director del Laboratorio de Conversión de Energías y Electrónica de Potencia, Universidad de Talca, Curicó, Chile.

Patrick Wheeler, Pericle Zanchetta. Power Electronics, Machines and Control Group, The University of Nottingham, Nottingham, UK.

Galina Mirzaeva. School of Electrical Engineering and Computing, The University of Newcastle, Newcastle, Australia.

Jaime Rohten, Universidad del Bío Bío, Concepción, Chile.

978-0-7381-3333-1/21\$31.00 @2021 IEEE

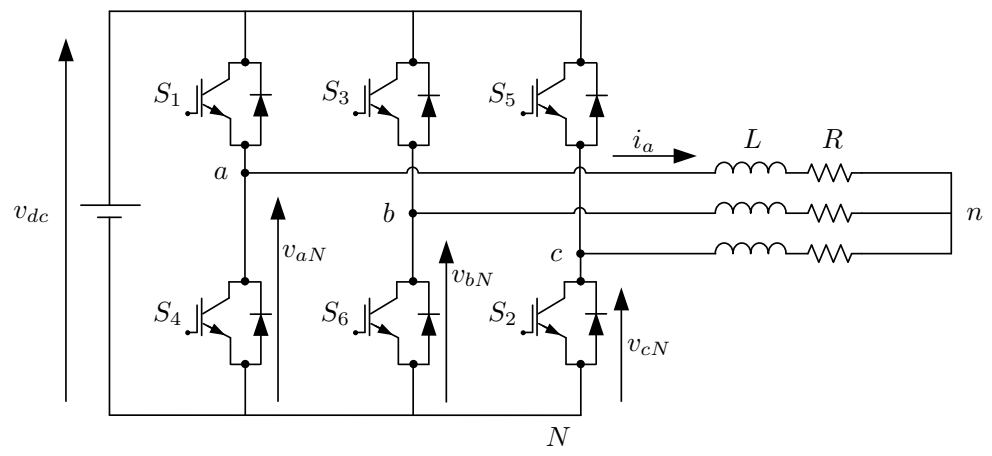

Fig. 1: Topología del inversor fuente de voltaje.

Tabla I: Estados válidos del VSI

\begin{tabular}{c|cccccc} 
Estado & $S_{1}$ & $S_{2}$ & $S_{3}$ & $S_{4}$ & $S_{5}$ & $S_{6}$ \\
\hline 1 & 1 & 1 & 0 & 0 & 0 & 1 \\
2 & 1 & 1 & 1 & 0 & 0 & 0 \\
3 & 0 & 1 & 1 & 1 & 0 & 0 \\
4 & 0 & 0 & 1 & 1 & 1 & 0 \\
5 & 0 & 0 & 0 & 1 & 1 & 1 \\
6 & 1 & 0 & 0 & 0 & 1 & 1 \\
7 & 1 & 0 & 1 & 0 & 1 & 0 \\
8 & 0 & 1 & 0 & 1 & 0 & 1
\end{tabular}

Tabla II: Voltajes línea-línea y corrientes del VSI

\begin{tabular}{c|cccc} 
Estado & $v_{a b}$ & $v_{b c}$ & $v_{c a}$ & $i_{d c}$ \\
\hline 1 & $v_{d c}$ & 0 & $-v_{d c}$ & $i_{a}$ \\
2 & 0 & $v_{d c}$ & $-v_{d c}$ & $i_{a}+i_{b}$ \\
3 & $-v_{d c}$ & $v_{d c}$ & 0 & $i_{b}$ \\
4 & $-v_{d c}$ & 0 & $v_{d c}$ & $i_{b}+i_{c}$ \\
5 & 0 & $-v_{d c}$ & $v_{d c}$ & $i_{c}$ \\
6 & $v_{d c}$ & $-v_{d c}$ & 0 & $i_{a}+i_{c}$ \\
7 & 0 & 0 & 0 & 0 \\
8 & 0 & 0 & 0 & 0
\end{tabular}

resultado, solo 8 estados de conmutación posibles son permitidos en este convertidor, con lo cual los voltajes de salida línea-línea y corriente en el enlace DC son generados (Tabla I y Tabla II). La corriente del enlace DC $i_{d c}$ se determina como una función de los semiconductores de conmutación del inversor y las corrientes de salida $\mathbf{i}$,

$$
i_{d c}=\left[\begin{array}{lll}
S_{1} & S_{3} & S_{5}
\end{array}\right] \mathbf{i}
$$

El voltaje de salida está sintetizado como una función de los semiconductores de conmutación del inversor y el voltaje del enlace DC $v_{d c}$, como se muestra en la ecuación (2). 


$$
\mathbf{v}=\left[\begin{array}{c}
S_{1} \\
S_{3} \\
S_{5}
\end{array}\right] v_{d c}
$$

En las siguientes secciones se presentan distintas funciones de costos para distintos objetivos en un control predictivo. El funcionamiento del control predictivo consiste en determinar en cada instante de muestreo el valor de cada función de costo dependiendo de los estados de conmutación del convertidor de potencia y escoger el estado de conmutación que produce el mínimo valor de la función de costo para ser aplicado en el instante siguiente. Además en las simulaciones sólo se considera $k+1$, esto quiere decir se considera un horizonte de predicción de 1. Sin embargo, a la hora de implementar el control predictivo de manera experimental, al menos se debe considerar un horizonte de predicción de $2(k+2)$ para compensar el retardo.

\section{Control para el Seguimiento de UnA REFERENCIA}

El objetivo de control consiste en que una variable controlada sigue una señal de referencia determinada. Entre los ejemplos comunes en los convertidores de potencia están el control de corriente, voltaje y potencia. La forma de representar estos términos son por medio del error absoluto (ecuación (3)), error al cuadrado (ecuación (4)) y la integral del error (ecuación (5)).

$$
\begin{gathered}
g=\left|x^{*}-x^{p}\right| \\
g=\left(x^{*}-x^{p}\right)^{2} \\
g=\left|\int_{k}^{k+1}\left(x^{*}(t)-x^{p}(t)\right) d t\right|
\end{gathered}
$$

El error absoluto y el error al cuadrado entregan los mismos resultados considerando un sólo término en la función de costo, sin embargo en una función con más términos, el error al cuadrado tiene mejores resultados [5]. Algunos ejemplos son el control de corriente en coordenadas ortogonales $\alpha \beta$ [6] (ecuación (6)), control de flujo y torque [7] (ecuación (7)), control de corriente con balance de voltaje de los condensadores [8] (ecuación (8)), control de potencia [9] (ecuación (9)) y seguimiento del máximo punto de potencia [10] (ecuación (10)).

$$
\begin{gathered}
g=\left|i_{\alpha}^{*}-i_{\alpha}^{p}\right|+\left|i_{\beta}^{*}-i_{\beta}^{p}\right| \\
g=\left|T_{e}^{*}-T_{e}^{p}\right|+\left.\lambda_{\psi}|| \psi\right|^{*}-|\psi|^{p} \mid \\
g=\left|\mathrm{i}^{*}-\mathrm{i}^{p}\right|+\left|v_{c 1}^{p}-v_{c 2}^{p}\right| \\
g=\left|P^{*}-P^{p}\right|+\left|Q^{*}-Q^{p}\right|
\end{gathered}
$$

$$
g=\lambda_{I}\left|I_{L}^{p}-I_{L}^{*}\right|+\lambda_{v}\left|V_{p v}^{p}-V^{*}\right|
$$

donde $i_{\alpha}^{p}$ es la corriente predictiva en la carga en coordenada $\alpha, i_{\beta}^{p}$ es la corriente predictiva en la carga en coordenada $\beta, i_{\alpha}^{*}$ es la corriente de referencia en coordenada $\alpha, i_{\beta}^{*}$ es la corriente de referencia en coordenada $\beta, T_{e}^{p}$ es el torque electromagnético predictivo, $\lambda_{\psi}$ es el factor de peso asociado al vector de flujo, $|\psi|^{p}$ es el vector de flujo predictivo, $T_{e}^{*}$ es el torque electromagnético de referencia, $|\psi|^{*}$ es el vector de flujo de referencia, $\mathrm{i}^{p}$ es el vector de corriente en la carga predictiva, $v_{c 1}^{p}$ es el voltaje del condensador $c 1$ predictivo, $v_{c 2}^{p}$ es el voltaje del condensador $c 2$ predictivo, $\mathrm{i}^{*}$ es el vector de corriente en la carga de referencia, $P^{p}$ es la potencia activa predictiva, $Q^{p}$ es la potencia reactiva predictiva, $P^{*}$ es la potencia activa de referencia, $Q^{*}$ es la potencia reactiva de referencia, $\lambda_{I}$ es el factor de peso de la corriente en el inductor, $I_{L}^{p}$ es la corriente predictiva en el inductor, $\lambda_{v}$ es el factor de peso del voltaje en el panel fotovoltaico, $V_{p v}^{p}$ es el voltaje predictivo en el panel fotovoltaico, $I_{L}^{*}$ es la corriente de referencia en el inductor y $V^{*}$ es el voltaje de referencia del panel fotovoltaico.

\section{Restricciones de Desempeño}

Las restricciones de desempeño son importantes para reducir el esfuerzo del control predictivo. En los convertidores de potencia, el esfuerzo del control es relacionado a las variaciones de corriente y voltaje, frecuencia de conmutación, o pérdidas por conmutación [5]. Las restricciones de desempeños son términos que se agregan a la función de costo. En un inversor fuente de voltaje, un esfuerzo de control puede ser representado por los cambios de los vectores de voltaje aplicados a una carga y es expresado como

$$
g=\left|i_{\alpha}^{*}-i_{\alpha}^{p}\right|+\left|i_{\beta}^{*}-i_{\beta}^{p}\right|+\lambda_{v}|| \mathrm{v}(k-1)-\mathrm{v}(k)||
$$

El término adicional en la ecuación (11) representa la diferencia entre el vector de voltaje aplicado previamente $\mathrm{v}(k-1)$ y el vector de voltaje que se aplica $\mathrm{v}(k)$ [5]. Para evaluar el desempeño del control, se realizó una simulación en Matlab/Simulink usando un inversor fuente voltaje trifásico con carga $R L$. Además se considera un rectificador de onda completa alimentado por una fuente de voltaje AC quien se encarga del control del voltaje en el enlace DC. Los parámetros del sistema son $V_{r m s}=220 \mathrm{~V}, R=10 \Omega, C=1,9 \mathrm{mF}$, $L=10 \mathrm{mH}$ y $T_{s}=10 \mu \mathrm{s}$. Como se puede apreciar en la Figura 2 a mayor valor del factor de peso $\lambda_{v}$ se reduce la cantidad de conmutaciones generadas en $v_{a n}$ pero aumenta la distorsión de la corriente $i_{a}$. El THD (distorsión armónica total) de la corriente correspondiente a los tres valores de $\lambda_{v}$ $(0.005,0.002$ y 0.0005$)$ corresponde a $20.5 \%, 8.25 \%$ y $2 \%$ respectivamente.

\section{Minimización de la Frecuencia de CONMUTACIÓN}

La frecuencia de conmutación es una medida importante de desempeño del control de un convertidor de potencia, por lo 


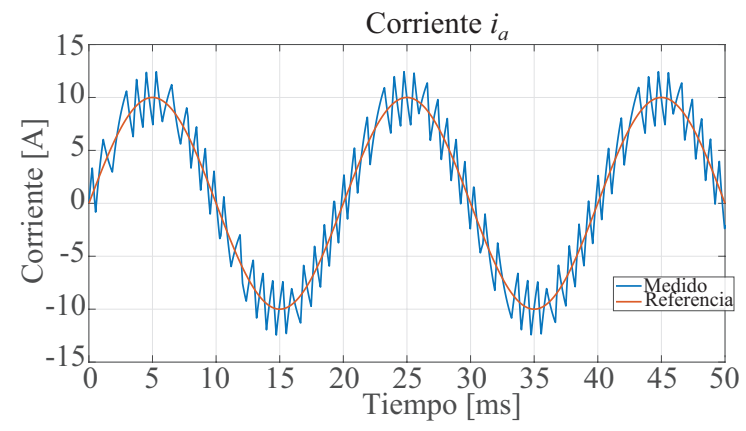

(a)

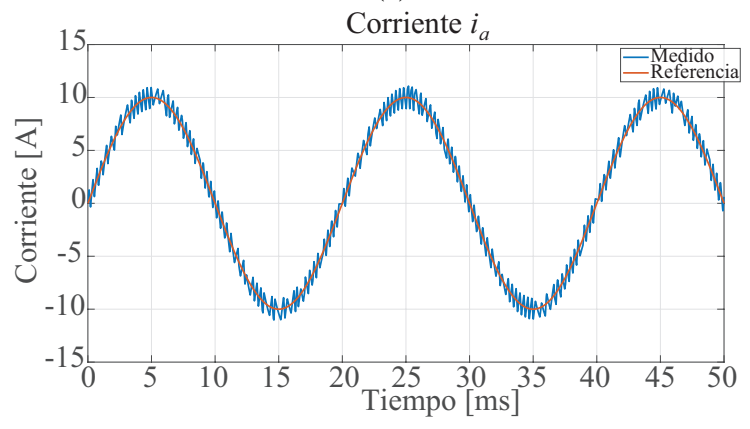

(c)

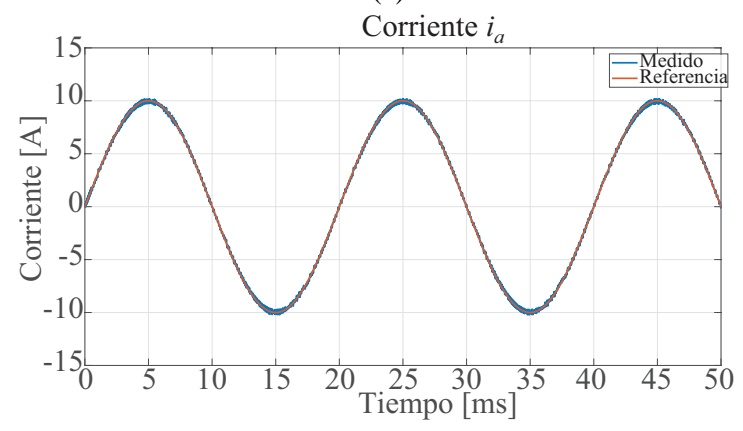

(e)

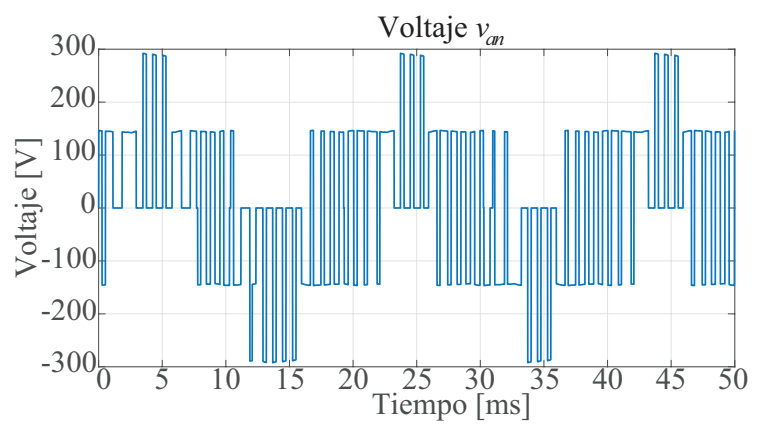

(b)

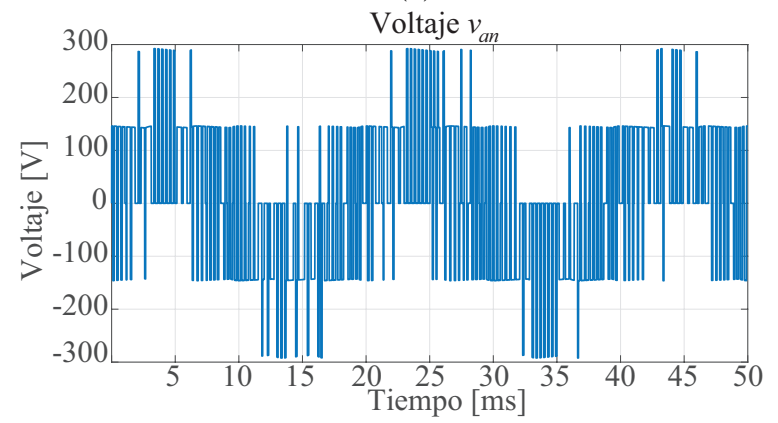

(d)

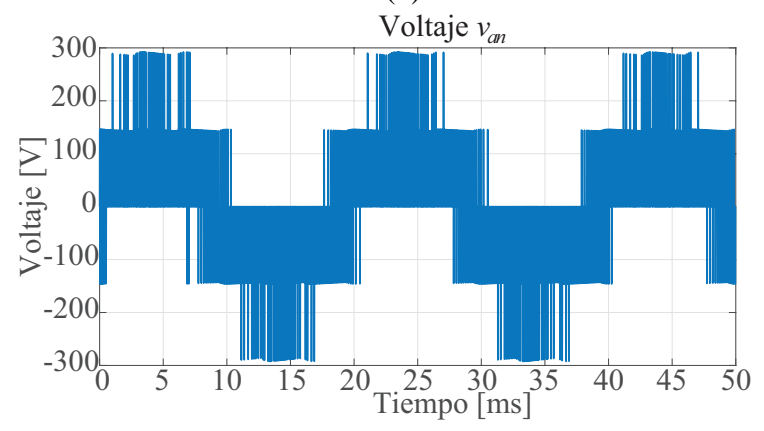

(f)

Fig. 2: Corriente $i_{a}$ y voltaje $v_{a n}$ para distintos valores de $\lambda_{v} \cdot \lambda_{v}=0,005$ (a) y (b), $\lambda_{v}=0,002(\mathrm{c}) \mathrm{y}(\mathrm{d}), \lambda_{v}=0,0005(\mathrm{e}) \mathrm{y}$ (f).

tanto reducir el número de conmutaciones es otro requerimiento. Esto consiste en incluir un término en la función de costo que cubre el número de conmutaciones, cuando se pasa a un estado de conmutación $\mathrm{S}(k)$ desde un estado de conmutación aplicado previamente $\mathrm{S}(k-1)$ [5].

Esto queda expresado como

$$
g=\left(i_{\alpha}^{*}-i_{\alpha}^{p}\right)^{2}+\left(i_{\beta}^{*}-i_{\beta}^{p}\right)^{2}+\lambda_{n} n
$$

donde $n$ es el número de conmutaciones que se realizan para pasar al estado de conmutación $\mathrm{S}(k)$. Para el caso de un VSI trifásico, $n$ queda expresado como

$n=\left|S_{a}(k)-S_{a}(k-1)\right|+\left|S_{b}(k)-S_{b}(k-1)\right|+\left|S_{c}(k)-S_{c}(k-1)\right|$

Como se puede ver en la Tabla III, al incrementar el factor de peso $\lambda_{n}$, aumenta el THD de la corriente $i_{a}$. Por otro lado, a modo de analizar la reducción del número de conmutaciones, se realiza un conteo en el intervalo de tiempo de 0 a $0.005 \mathrm{~s}$ de los cambios de estado de cada switch y luego se promedia para cada valor de $\lambda_{n}$ como se presenta en la Tabla III. Se puede mencionar que a medida que aumenta el valor del factor de peso $\lambda_{n}$ se reduce la cantidad de conmutaciones.

En la Figura 3 se pueden apreciar las formas de onda de $i_{a}$ y $v_{a n}$ para dos valores de $\lambda_{n}$, cuyo valor más grande produce menores conmutaciones y mayor distorsión en la corriente $i_{a}$.

Tabla III: Distorsión armónica total para cada $\lambda_{n}$ y el promedio de cambios de estado de los switches.

\begin{tabular}{c|c|c}
$\lambda_{n}$ & THD de $i_{a}$ & $\mathrm{~N}^{\circ}$ cambios estado switch(promedio) \\
\hline 1.8 & $24.20 \%$ & 5 \\
1.2 & $17.38 \%$ & 6 \\
0.4 & $6.69 \%$ & 18 \\
0.09 & $2.08 \%$ & 57
\end{tabular}




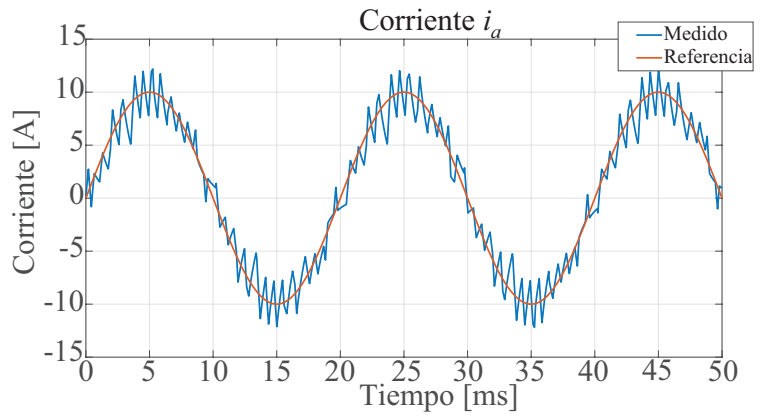

(a)

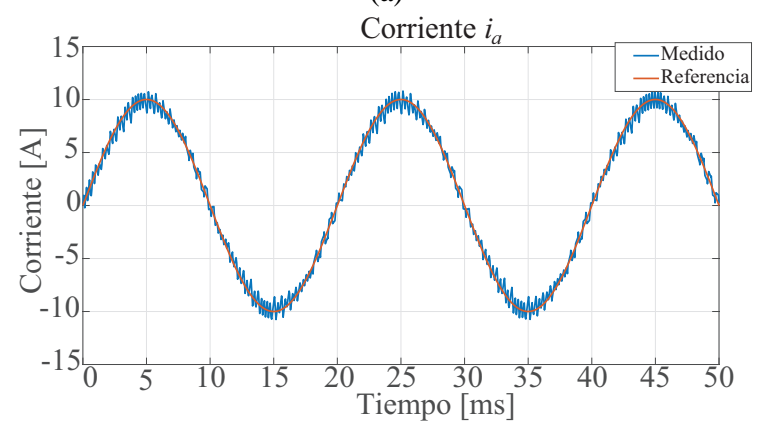

(c)

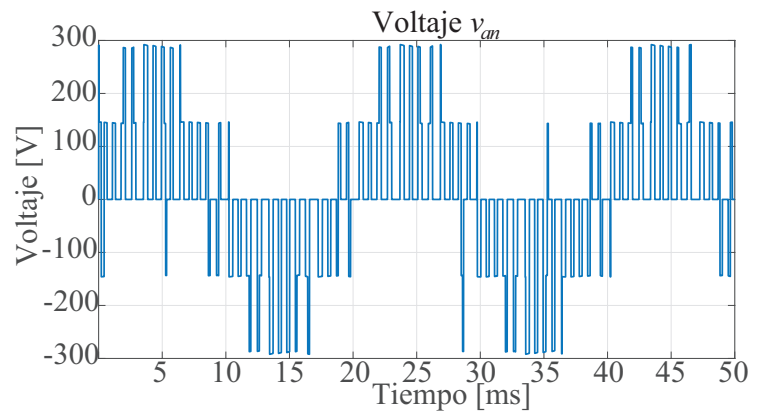

(b)

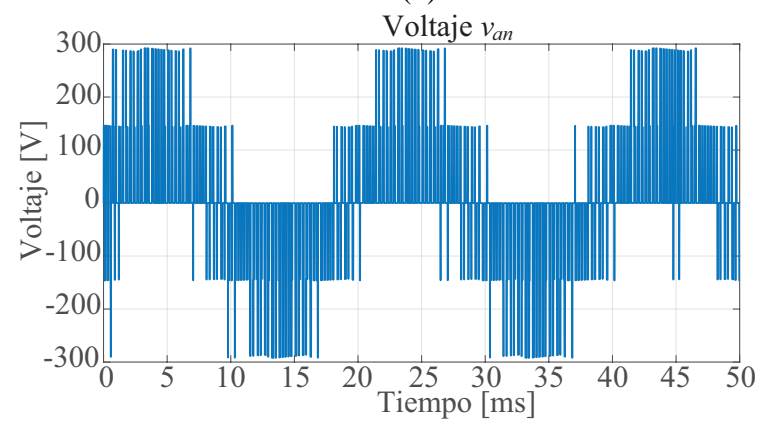

(d)

Fig. 3: Corriente $i_{a}$ y voltaje $v_{a n}$ para distintos valores de $\lambda_{n}$. $\lambda_{n}=1,2$ (a) y (b), $\lambda_{n}=0,4$ (c) y (d).

\section{RESTRICCIONES ESTRICTAS}

Otra de las ventajas del control predictivo es que se pueden agregar distintas restricciones para limitar directamente ciertos valores de corriente, voltaje o potencia.

Un ejemplo es la de evitar que algunas corrientes de pico en la carga de un VSI trifásico supere cierto valor, producto de la conmutación de los semiconductores de potencia. La función de costo queda expresada como

$$
g=\left(i_{\alpha}^{*}-i_{\alpha}^{p}\right)^{2}+\left(i_{\beta}^{*}-i_{\beta}^{p}\right)^{2}+f_{\text {lim }}\left(i^{p}\right)
$$

donde $f_{\text {lim }}\left(i^{p}\right)$ es una función que condiciona los peaks de corriente en la carga, y queda expresada como

$$
f_{l i m}\left(\mathrm{i}_{\alpha \beta}{ }^{p}\right)=\left\{\begin{array}{lrr}
\infty & \text { si } & -i_{\max }>\mathrm{i}_{\alpha}^{p}\left(o \mathrm{i}_{\beta}^{p}\right)>i_{\max } \\
0 & & \text { en caso contrario }
\end{array}\right.
$$

$i_{\max }$ es la corriente máxima con un valor de $20 \mathrm{~A}$. Se evaluó un control predictivo con restricciones en un VSI trifásico con un tiempo de muestreo de $100 \mu \mathrm{s}$. En la Figura 4c se presenta la corriente $i_{a}$ donde por efectos de la conmutación, la señal tiene picos mayores de 20 A (Figura 4d ). En cambio al aplicar las restricciones, limita esta corriente en la carga de manera que no sobrepase los $20 \mathrm{~A}$ (Figuras 4a y 4b).

\section{RESTRICCIÓN EN EL CONTENIDO ARMÓNICO}

Otro de los requerimientos es regular o controlar el contenido armónico que tienen ciertas variables, producto de los efectos de la conmutación de los semiconductores de potencia.
En [5] se menciona que el contenido armónico es más distribuido al disminuir la frecuencia de muestreo. Por lo tanto, la frecuencia de conmutación es variable en un control predictivo. En algunas aplicaciones, tales espectros de frecuencia no son deseables porque pueden producir oscilaciones y hacen que el diseño de los filtros análogos sea complicado.

Por lo tanto, en un control predictivo se puede agregar un filtro digital en la función de costo para contrarrestar esta desventaja. La función de costo en [5] queda expresada como

$$
g=\left|F\left(i_{\alpha}^{*}-i_{\alpha}^{p}\right)\right|+\left|F\left(i_{\beta}^{*}-i_{\beta}^{p}\right)\right|
$$

donde $F$ es un filtro digital expresado como la función de transferencia

$$
F(z)=\frac{z^{0}+b_{1} z^{-1}+\ldots+b_{n} z^{-1}}{a_{0} z^{0}+a_{1} z^{-1}+\ldots+a_{n} z^{-n}}
$$

Para la evaluación, se diseñaron filtros digitales Butterworth rechazo banda de cuarto orden, con frecuencias de $2 \mathrm{kHz}$ y $4 \mathrm{kHz}$. Para seleccionar los valores de los parámetros de $b$ y $a$ en una determinada banda de rechazo se usa el comando 'butter' tal como se presenta en el siguiente código:

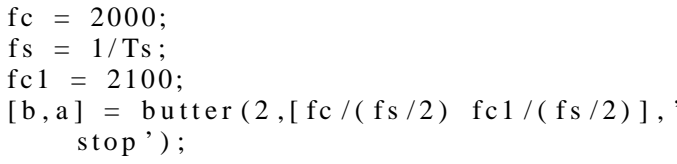

donde $f c$ y $f c 1$ son las frecuencias del rango de la banda de rechazo, $f s$ es la frecuencia de muestreo y 'stop' es el 


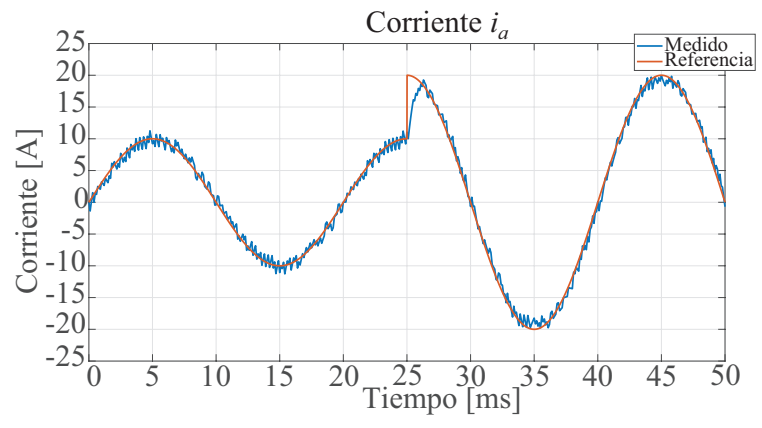

(a)

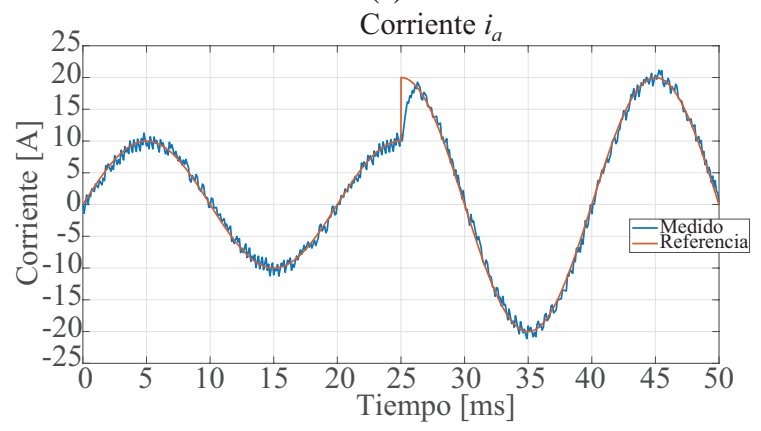

(c)

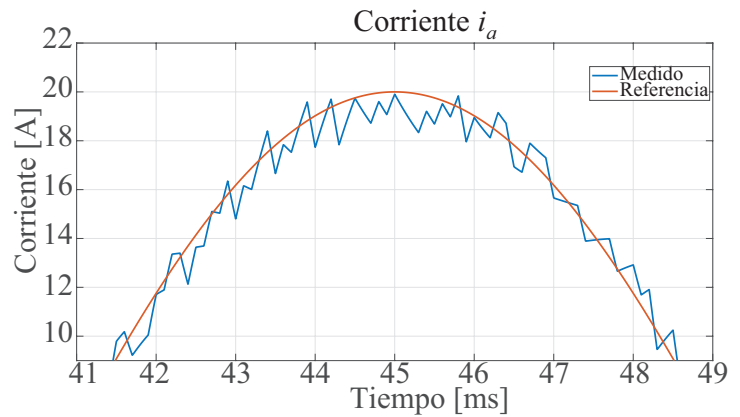

(b)

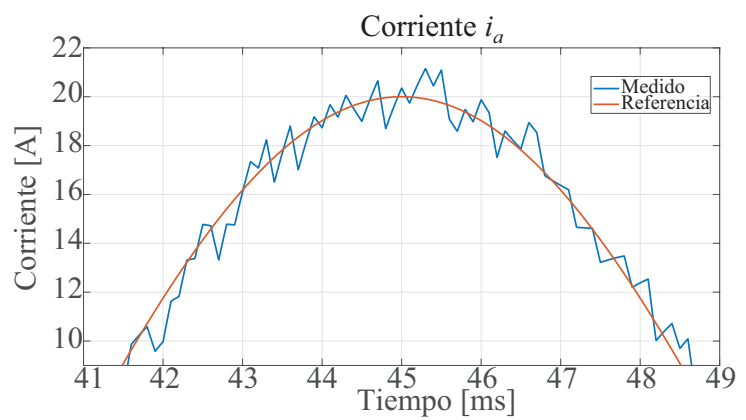

(d)

Fig. 4: (a) Corriente $i_{a}$ con limitación, (b) zoom corriente $i_{a}$ con limitación, (c) corriente $i_{a}$ sin limitación y (d) zoom corriente $i_{a}$ sin limitación.

comando que determina que es un filtro digital de rechazo banda. Luego obtenida la función de transferencia en el plano $\mathrm{z}$, se realiza la transformación a diferencias finitas para ser implementado en el control. Los resultados de simulación se presentan en la Figura 5 para el caso de frecuencia de $2 \mathrm{kHz}$ y en la Figura 7 para el caso de $4 \mathrm{kHz}$.

Ambas formas de onda de la corriente se presentan en la Figura 5a y 7a. En el espectro armónico de la Figura 5b, se puede apreciar que se concentra cerca de la frecuencia de corte del filtro diseñado, que en este caso es de $2 \mathrm{kHz}$ y ocurre lo mismo en el espectro la Figura $7 \mathrm{~b}$ en los $4 \mathrm{kHz}$. Gracias al filtro implementado es posible concentrar el espectro armónico en una frecuencia deseada, evitando armónicos contenidos en un rango de frecuencias [5]. La respuesta en frecuencia de los filtros digitales diseñados de $2 \mathrm{kHz}$ y $4 \mathrm{kHz}$, se presentan en las Figuras 6 y 8 respectivamente.

\section{CONCLUSIONES}

En este artículo se presentaron diferentes formas de implementar una función de costo. También se presentaron distintos términos en la función de costo para controlar diferentes requerimientos en un convertidor de potencia fuente de voltage. Entre los distintos requerimientos evaluados en un VSI trifásico, se consideró el seguimiento a las corrientes $\alpha \beta$ de referencia, minimización del número de vectores de voltaje, minimización del número de conmutaciones y pérdidas, limitación del valor de la corriente en la carga y control en el contenido armónico.

Una de las ventajas del control predictivo es la simplicidad de implementar múltiples objetivos con solo adicionar térmi- nos en la función de costo, dependiendo de los requerimientos del diseño. Sin embargo esto conlleva al aumento del tiempo de ejecución del control predictivo, por lo tanto al momento del diseño de la función de costo, se debe agregar los objetivos más importantes que permiten mejorar el desempeño del convertidor de potencia considerando la carga computacional.

Desde el punto de vista de la implementación experimental, se debe considerar un horizonte de predicción de al menos 2 para compensar el retardo de medición y procesamiento, como también se debe considerar los efectos de los errores en los valores de los parámetros del sistema, inexactitud de los sensores, ruido electromagnético, corrientes y capacitancias parásitas, tiempo de ejecución del algoritmo de predicción, velocidad de procesamiento del controlador, entre otros aspectos.

Por otro lado, se puede concluir que la forma de encarar el equilibrio entre los distintos objetivos de control para una misma aplicación, que consiste en incluirlos todos en una sumatoria, convierte el problema multiobjetivo en uno monoobjetivo, y de esta forma permite un cálculo más rápido, dependiendo directamente del factor de peso otorgado a las distintas funciones y puede dar lugar a soluciones no óptimas en un sentido multiobjetivo estricto.

\section{AGRADECIMIENTOS}

Los autores agradecen el apoyo económico del Proyecto de Investigación FONDECYT Regular 1191028, MEC 80190074, MEC 801800974, ANID Becas/Doctorado Nacional 21201878 y FONDAP SERC Chile 15110019. 


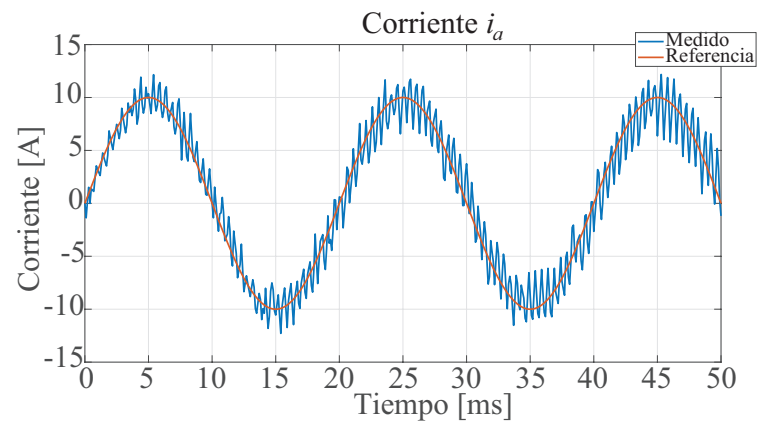

(a)

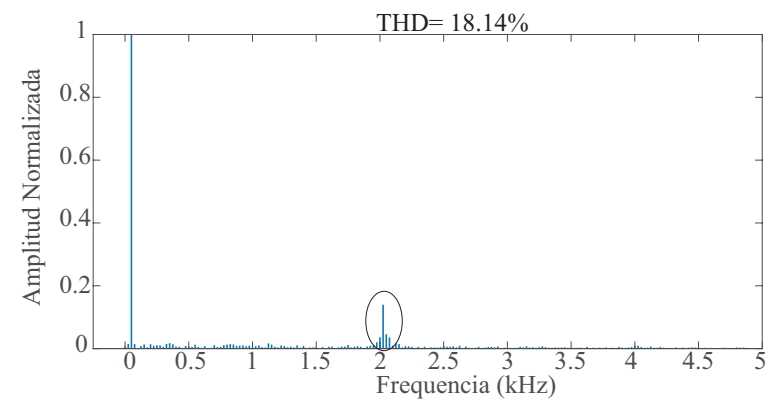

(b)

Fig. 5: (a) Corriente $i_{a}$, (b) contenido armónico corriente $i_{a}$.
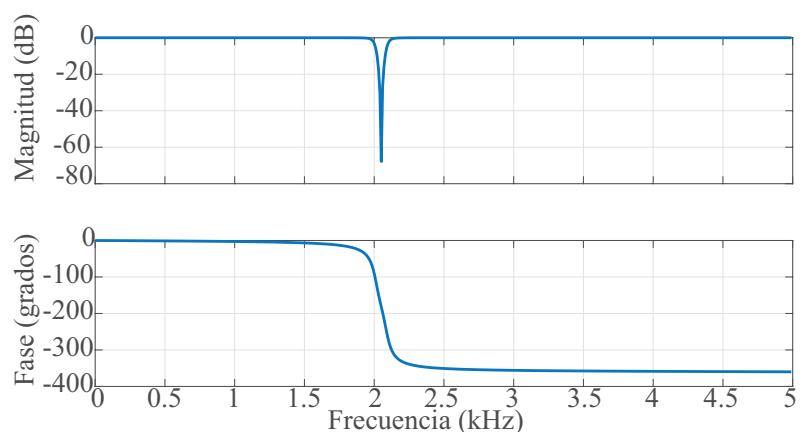

Fig. 6: Respuesta en frecuencia de un filtro digital Butterworth rechaza banda $(2 \mathrm{kHz})$.

\section{REFERENCIAS}

[1] F. Mwasilu, E. Kim, M. S. Rafaq, and J. Jung, "Finite-set model predictive control scheme with an optimal switching voltage vector technique for high-performance ipmsm drive applications," IEEE Transactions on Industrial Informatics, vol. 14, no. 9, pp. 3840-3848, 2018.

[2] Z. Zheng, D. Sun, M. Wang, and H. Nian, "Model predictive control with a novel cost function evaluation scheme for ow-pmsm drives," Electronics Letters, vol. 56, no. 13, pp. 655-657, 2020.

[3] M. B. Shadmand, S. Jain, and R. S. Balog, "Autotuning technique for the cost function weight factors in model predictive control for power electronic interfaces," IEEE Journal of Emerging and Selected Topics in Power Electronics, vol. 7, no. 2, pp. 1408-1420, 2019.

[4] J. Hang, H. Wu, J. Zhang, S. Ding, Y. Huang, and W. Hua, "Cost function-based open-phase fault diagnosis for pmsm drive system with model predictive current control," IEEE Transactions on Power Electronics, pp. 1-1, 2020.

[5] Cost Function Selection. John Wiley \& Sons, Ltd, 2012, ch. 10, pp. 145-161. [Online]. Available: https://onlinelibrary.wiley.com/doi/abs/10.1002/9781119941446.ch10

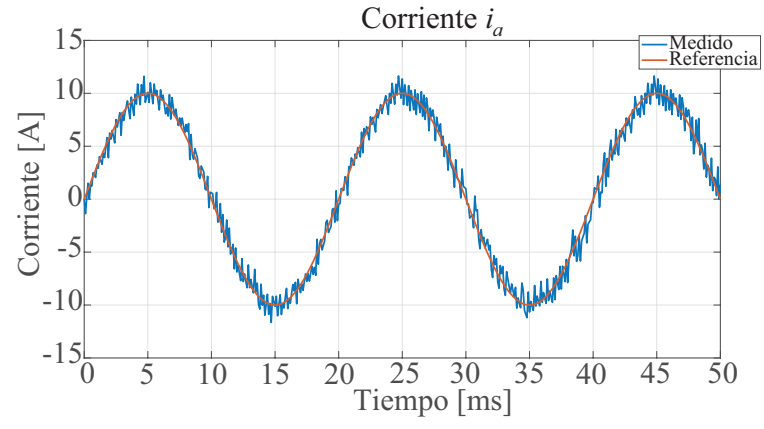

(a)

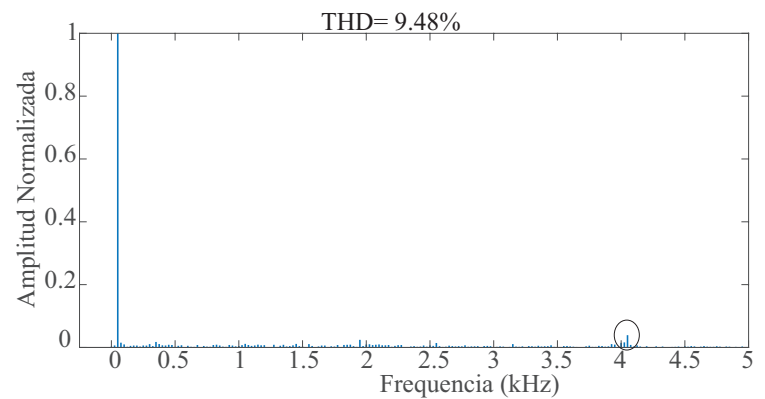

(b)

Fig. 7: (a) Corriente $i_{a}$, (b) contenido armónico corriente $i_{a}$.

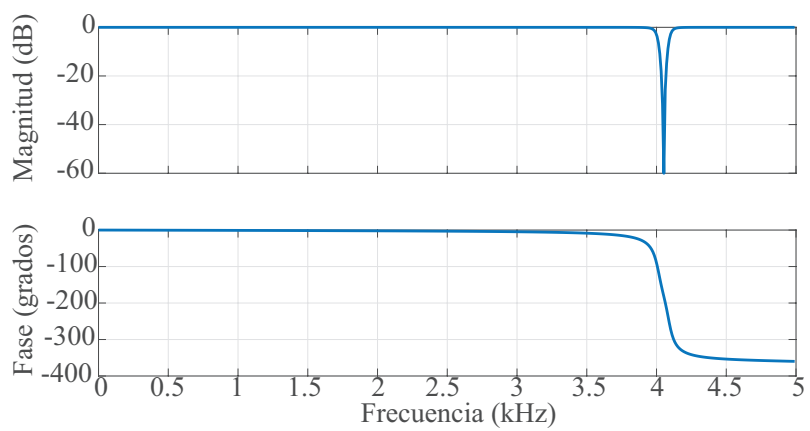

Fig. 8: Respuesta en frecuencia de un filtro digital Butterworth rechaza banda $(4 \mathrm{kHz})$.

[6] F. Blaabjerg, Control of Power Electronic Converters and Systems Vol 1, 022018.

[7] Predictive Control of Induction Machines. John Wiley \& Sons, Ltd, 2012, ch. 8, pp. 115-132. [Online]. Available: https://onlinelibrary.wiley.com/doi/abs/10.1002/9781119941446.ch8

[8] Predictive Control of a Three-Phase Neutral-Point Clamped Inverter. John Wiley \& Sons, Ltd, 2012, ch. 5, pp. 65-79. [Online]. Available: https://onlinelibrary.wiley.com/doi/abs/10.1002/9781119941446.ch5

[9] Control of an Active Front-End Rectifier. John Wiley \& Sons, Ltd, 2012, ch. 6, pp. 81-98. [Online]. Available: https://onlinelibrary.wiley.com/doi/abs/10.1002/9781119941446.ch6

[10] A. Lashab, D. Sera, J. M. Guerrero, L. Mathe, and A. Bouzid, "Discrete model-predictive-control-based maximum power point tracking for pv systems: Overview and evaluation," IEEE Transactions on Power Electronics, vol. 33, no. 8, pp. 7273-7287, 2018. 